\title{
Moins de rentes - davantage de coûts! La prévoyance professionnelle sous les feux de la rampe!
}

Quelles sont les raisons pour lesquelles les coûts pour les risques et les frais administratifs augmentent dans les institutions de prévoyance collectives alors que les taux de conversion des rentes sont abaissés? Chance pour notre institution de prévoyance PAT-LPP propre à la profession? Interview, par Mme Annamaria Müller Imboden (AMI), Secrétaire générale de la FMH et membre du conseil de la fondation de prévoyance PAT-LPP, de M. Eugen Rieder (ER), directeur de la PAT-LPP, Fondation de prévoyance pour le personnel des médecins et des vétérinaires. suisse AVS AI qui gère les comptes de nos membres et profite des effets de synergie se révèle payante. A cela s'ajoute que nous sommes en mesure d'offrir une prime de risque plus avantageuse grâce à l'évolution favorable des sinistres dans notre cercle d'assurés (statistiquement très en dessous des prévisions).

AMI: Le taux de conversion des rentes doit être abaissé de $19 \%$ pour les hommes et même de $24 \%$ pour les femmes. Quelles en sont les conséquences? ER: Si le taux de conversion des rentes des institutions collectives de prévoyance devait passer de 7,2\% actuellement à 5,8\% (hommes) et 5,4\% (femmes), cela aura pour conséquence qu'un capital-vieillesse de Fr. 1 million au début de la retraite, au lieu de générer une rente annuelle de Fr. 72000.- comme aujourd'hui ne donnera plus droit qu'à une rente annuelle de Fr. 58000.-, resp. de Fr. 54000.-.

AMI: Quelles hypothèses et quelles données fondamentales justifient-elles ces valeurs basses?

ER: Les données exactes n'ont pas (encore) été publiées. En essayant de bien comprendre ces chiffres nous sommes parvenus, avec des spécialistes, à la conclusion que l'on est parti dans tous les domaines de l'évolution la plus défavorable possible: coûts administratifs continuant d'augmenter, persistance de l'augmentation des cas d'invalidité, rendement futur des placements situé entre $2 \%$ et $2,5 \%$, espérance de vie continuant une progression sans frein.

AMI: Et sur quelles valeurs repose l'appréciation à laquelle a procédé la PAT-LPP?

ER: Nous tablons à long terme sur un rendement moyen de nos placements d'au moins 3,5\% ainsi que sur une légère augmentation des cas d'invalidité. Nous ne nous attendons néanmoins pas à une progression sans frein de l'espérance de vie. La statistique 2000 permet déjà de discerner, pour les femmes, des signes de «stagnation».

\section{AMI: D'où proviennent ces différences?}

ER: La raison de ces différences réside dans les coûts administratifs et les primes de risques qui sont notablement plus élevées dans les institutions collectives de prévoyance. Notre collaboration avec la Caisse de compensation medi-
AMI: Certaines institutions collectives de prévoyance ont déjà mis en place un droit de résiliation pour les assurés, les travailleurs indépendants et les institutions pour le 31 décembre 2003 ou ont 
même déjà dénoncé le contrat. Que recommande la PAT-LPP aux personnes ainsi touchées?

ER: Nous recommandons de résilier les contrats pour le 31 décembre 2003 à titre de précaution, mais sous réserve de révocation, de demander des offres à la concurrence et de décider ensuite. Pourquoi avec réserve de révocation? Afin de ne subir aucune diminution de prestation dans le domaine supra-obligatoire, en cas de maladie préexistante.

AMI: On peut s'attendre à ce qu'un grand nombre de demandes parviennent à la PAT-LPP. L'administration pourra-t-elle maîtriser un tel flux?

ER: Nos nouveaux plans d'assurances ont généré l'année passée une croissance de $37 \%$. Grâce à la gestion administrative simplifiée, nous pouvons «digérer» encore davantage, en étant toutefois au maximum de nos disponibilités.

AMI: Du point de vue administratif, sans doute, mais comment cela se présente-t-il en ce qui concerne les prestations de conseils de la PAT-LPP? ER: Les personnes intéressées peuvent s'adresser aux conseillers spécialisés. Il est ensuite important de procéder à des comparaisons dans le domaine des coûts administratifs et des primes de risques. S'agissant du calcul du capital au moment de l'entrée à la retraite, tous opèrent les calculs avec le même taux d'intérêt, toutefois par exemple à partir de l'âge de 55 ans, avec des contributions d'épargne plus élevées. Par voie de conséquence, le capital-vieillesse au moment de l'âge de la retraite ne constitue pas une réelle donnée chiffrée susceptible d'être comparée. En procédant à une comparaison, la concurrence a maintes fois dissimulé les «non-primes d'épargne» élevées.

AMI: Existe-t-il une collaboration entre la PAT-LPP et la FMH Insurance \& Financial Services SA?

\begin{tabular}{|c|c|c|c|c|}
\hline & $\begin{array}{l}\text { Nombre } \\
\text { d'assurés }\end{array}$ & $\begin{array}{l}\text { Revenus } \\
\text { assurés } \\
\text { Mio. Fr. }\end{array}$ & $\begin{array}{l}\text { Cotisations } \\
\text { annuelles } \\
\text { Mio. Fr. }\end{array}$ & $\begin{array}{l}\text { Avoir des } \\
\text { membres } \\
\text { Mio. Fr. }\end{array}$ \\
\hline 31.12 .1999 & 3898 & 145 & 20,58 & 134,51 \\
\hline 31.12 .2000 & 4020 & 144 & 21,93 & 165,24 \\
\hline 31.12 .2001 & 4292 & 194 & 28,54 & 210,28 \\
\hline 31.12 .2002 & 4910 & 255 & 37,62 & 291,89 \\
\hline Depuis $31.12 .99+$ & $26,0 \%$ & $76,2 \%$ & $82,8 \%$ & $117,0 \%$ \\
\hline
\end{tabular}

ER: Nous bénéficions d'un excellent soutien de la part de cet office de conseils spécialisés sur les besoins des médecins et considérons comme une de nos tâches principales de soutenir et de promouvoir cette collaboration - comme aussi avec d'autres firmes bien introduites - grâce à un service diligent et des échanges d'informations.

AMI: Le rapport annuel 2002 de la PAT-LPP vient d'être distribué; quelles réactions avez-vous enregistrées à ce propos?

ER: Sans exception: positives. Il a été estimé bien fondé que nous n'ayons pas abandonné le marché des actions, que nous ayons choisi avec beaucoup d'attention des placements dans des «hedge fonds», que nous veillions à diversifier nos investissements afin qu'il en résulte une bonne performance à long terme ainsi que nous ayons recherché des spécialistes indépendants pour nous conseiller et accompagner nos pas. Les discussions menées à ce sujet depuis le printemps ont confirmé ce jugement positif.

Le taux de couverture de 90,8\% a soulevé à nouveau la question de savoir si les employeurs devaient apporter maintenant une contribution supplémentaire ou si nous devions reporter à plus tard l'examen des demandes d'avances servant à financer l'accès à la propriété immobilière. Ces deux éventualités ne sont pas de saison. En effet, grâce à notre croissance élevée nous nous trouvons, malgré l'insuffisance de couverture, dans une situation très favorable.

AMI: Qu'entreprend la PAT-LPP pour améliorer le taux de couverture?

ER: Il est prévu, à partir de 2004, de mettre à la charge des assurés une partie du montant des coûts administratifs qui avaient été supportés jusqu'ici par la caisse. En outre, dans l'intérêt à long terme de notre fondation, un léger ajustement des primes de risques est prévu. Dès lors que nous connaissons une forte croissance persistante, le conseil de fondation ne décidera de l'augmentation qu'en novembre, car nous n'aimerions pas charger nos assurés plus que ce dont nous avons besoin. Nous pouvons de la sorte garantir que l'excellent rapport prix/prestations pourra aussi être maintenu à l'avenir.

Il est important, en matière de taux de couverture, de considérer que:

- le déficit de couverture est purement conjoncturel;

- les bénéfices actuariels (moins de cas d'invalidité) demeurent chaque année en caisse et que d'éventuelles «mauvaises» années peuvent être couvertes par une réassurance de type «stop loss». 
Le facteur le plus important reste le rendement des placements par rapport au taux de rémunération LPP. La performance à mi-année est heureusement d'environ 3,3\% et, si le marché se maintient, nous pouvons tabler pour le 31 décembre 2003 sur une amélioration du taux de couverture, ce dernier étant aussi influencé très favorablement par notre forte croissance.
AMI: Tout cela résonne comme des perspectives «tout en rose» pour la PAT-LPP?

ER: Oui! Le contexte général pour notre fondation, qui s'engage à fond pour ses membres, sera toujours plus favorable. Nous sommes très bien armés avec les solutions d'assurance flexibles que nous offrons, comme les chiffres de notre évolution l'attestent.

AMI: Eugen Rieder, mille mercis pour ces informations claires et bien documentées.

\section{Séminaires / Seminari}

Bien qu'environ $40 \%$ de tous les médecins ouvrent tôt ou tard leur propre cabinet médical et ont besoin de connaissances en gestion d'entreprise hormis leur savoir-faire médical, cette thématique n'est souvent pas ou peu abordée. FMH Services qui s'occupe de la gestion d'entreprise des membres FMH comble désormais cette lacune en proposant des séminaires spécifiques. Dans ces séminaires, les médecins peuvent acquérir les bases nécessaires pour ouvrir leur propre cabinet médical ou un cabinet de groupe ou pour remettre leur cabinet de manière optimale. Les médecins obtiennent également des conseils sur la manière de collaborer avec les autorités ou avec des spécialistes tels que des experts-comptables, des conseillers financiers, en assurance ou en crédits bancaires, des juristes, des architectes, etc. Ces différents thèmes sont abordés par des experts qualifiés de la FMH et de FMH Services.

\section{Ouverture et gestion d'un cabinet médical}

\section{Participants}

Le séminaire est destiné aux médecins sur le point d'ouvrir ou de reprendre un cabinet médical.

\section{Contenu}

La réalisation d'un cabinet médical ne s'improvise pas. Vous allez dépendre du financement et de la gestion de votre cabinet médical. Ce séminaire traitera de l'environnement socio-politique qui sera le vôtre en tant qu'indépendant, et vous permettra de vous y préparer. Les thèmes suivants seront développés lors de ce séminaire: l'aménagement, le financement, les assurances, la fiscalité, la comptabilité et l'administration de votre cabinet.

\section{Documentation}

Basé sur une check-liste pour l'ouverture d'un cabinet médical, le classeur remis à chaque participant contient toutes les informations importantes.

\section{Frais / Sponsors}

Le séminaire est gratuit pour les membres FMH. Les frais sont couverts par les sponsors suivants: Caisse-Maladie des Médecins Suisses, Axon Lab SA et AstraZeneca SA

\section{Date}

No 19 Jeudi 18 septembre 2003 Hostellerie Genève $\quad 9.00-17.00 \mathrm{~h}$ de la Vendée

\section{Inscription}

Vous pouvez vous inscrire à l'adresse mentionnée ci-dessous ou via internet: www.fmhservices.ch.

\section{Remise/reprise d'un cabinet médical}

\section{Participants}

Le séminaire s'adresse aux médecins désirant remettre ou reprendre un cabinet médical. 